Original Research Article

\title{
Oral antifungal: the safety and efficacy of oral itraconazole in dermatophytosis
}

\author{
Vaishali Thakare ${ }^{1}$, Nandita K. Patel ${ }^{2}$, Sharmila Patil ${ }^{2}$, Navin Modi $^{3}$
}

\begin{abstract}
${ }^{1}$ Department of Pharmacology,
${ }^{2}$ Department of Dermatology, Venerology and Leprosy, Dr. D Y Patil Hospital, School of Medicine, Nerul, Navi Mumbai, Maharashtra, India

${ }^{3}$ Consultant Dermatologists, Mumbai, Maharashtra, India
\end{abstract}

Received: 29 August 2019

Revised: 04 October 2019

Accepted: 05 October 2019

*Correspondence to:

Dr. Nandita K. Patel,

Email: drnanditapatel@ gmail.com

Copyright: (C) the author(s), publisher and licensee Medip Academy. This is an openaccess article distributed under the terms of the Creative Commons Attribution NonCommercial License, which permits unrestricted noncommercial use, distribution, and reproduction in any medium, provided the original work is properly cited.

\begin{abstract}
Background: The systemic antifungals like Griseofulvin, Itraconazole, Terbinafine, Ketoconazole and Fluconazole are widely used for superficial fungal infection. Hepatotoxicity with oral antifungals is well established fact. The rate of transient asymptomatic changes in liver function tests accounts for about $0.5-10 \%$ of all patients treated with systemic antifungals. Clinical hepatic toxicity is seen less frequently. The aim of this study is to evaluate the effect of oral Itraconazole on hepatic function and it's efficacy in patients with extensive dermatophytosis.

Methods: The total of 524 patients with extensive dermatophytosis were included in our study which was conducted in a tertiary care hospital in Navi Mumbai.

Results: Itraconazole, a systemic antifungal agent is efficiently used in treatment of superficial and deep mycoses. It inhibits fungal cytochrome P450 dependent enzyme and thus impaires conversion of lanosterol to ergosterol. Adverse reactions to itraconazole includes drug reactions, gastrointestinal upset, headache, dizziness, thrombocytopenia, gynecomastia, reversible edema of extremities and metabolic side effects like hypokalemia, and hypertriglyceridemia. The level of hepatic transaminases increases in about 1\%$5 \%$ of patients who have received continuous therapy with systemic itraconazole. Clinical hepatitis rarely occurs in patients and, recovery generally ensues with the cessation of medication.

Conclusions: The baseline and post treatment liver function test is important to monitor if patient is on higher dose and longer duration of itraconazole therapy. The screening for high risk patients like poor liver function test, history of alcoholism, history of liver disease should be taken before stating the therapy.
\end{abstract}

Keywords: Itraconazole, Anti fungals, Hepatotoxicity

\section{INTRODUCTION}

There is a menace of dermatophytosis growing nowadays in India with changing clinical presentation and high recurrence rate. Superficial mycoses accounts for about $20-25 \%$ of all the infections in the world out of which dermatophytosis accounts for most of it. ${ }^{1}$ The systemic antifungals like griseofulvin, itraconazole, terbinafine, ketoconazole and fluconazole are widely used for superficial fungal infection. Hepatotoxicity with oral antifungals is a well-established fact. Most of the available data relate to ketoconazole and itraconazole, amongst which itraconazole is more effective and safer than ketoconazole. Since 1983, Itraconazole has been subjected thorough various clinical evaluation for safety and efficacy of the drug. It has been effectively used in treating patients of superficial mycoses including oral and vaginal candidiasis, dermatophytosis, and even in systemic mycoses. ${ }^{2}$ The most frequently encountered adverse events are gastrointestinal disturbance (nausea, constipation, abdominal pain) and drug reactions. The rate of transient asymptomatic changes in liver function tests accounts for about $0.5-10 \%$ of all the patients treated with systemic antifungals. Clinical hepatic toxicity is 
seen less frequently. ${ }^{3}$ There were controversial reports regarding the incidence of liver injury with itraconazole.

Aim of this study is to evaluate the effect of oral Itraconazole on hepatic function and its efficacy in extensive dermatophytosis. Primary objective is to evaluate the effect of oral Itraconazole on hepatic function and to evaluate the efficacy of Itraconazole. The secondary parameter is to evaluate the secondary bacterial infection in patients with extensive dermatophytosis, to evaluate the allergic association in extensive dermatophytosis and to evaluate association of random blood sugar level and dermatophytosis.

\section{METHODS}

\section{Study population}

All the patients suffering from extensive dermatophytosis attending the OPD of the Dr. D.Y. Patil Hospital in Navi Mumbai over a period of 1 year from December 2014December 2015. The term extensive dermatophytosis refers to patients suffering from tinea infection of more than 2 sites and the clinical lesions greater than $5 \mathrm{cms}$ in diameter.

\section{Inclusion criteria}

- Patients of both the sexes belonging to age group: 12-80 years.

- Newly diagnosed case of extensive dermatophytosis.

- The patients who are ready to be a part of study.

\section{Exclusion criteria}

- Age group : $<12$ years and $>80$ years

- Patients on anti-fungal therapy.

- Other than extensive tinea.

\section{Study centre and sampling method}

It is a prospective interventional study conducted in outpatient department of dermatology of a tertiary care teaching hospital, in a patient suffering from extensive tinea.

\section{Study procedure and duration}

Visit 1: Informed consent of all the patients suffering from extensive dermatophytosis was taken. Then the patients were subjected to routine dermatological examination along with detailed history. After taking the proper history, the patient's blood was collected for routine investigations (LFT, RBS, CBC) at the baseline visit. The data collected from the patients were noted down in the case record form and then entered in the excel sheet. Followed by that, the patients were prescribed oral Itraconazole $100 \mathrm{mg}$ twice daily for 6 weeks. The data was collected was on following parameters: Demographic details, severity of disease and treatment history.

Visit 2: After 3 weeks, the patients were called to see the effectiveness of the treatment.

Visit 3: After 6 weeks of course with oral Itraconazole, the patients were subjected to blood investigations to compare the baseline LFT with that after the completion of the study.

Descriptive statistics like mean, standard deviations and percentage proportions were used to describe baseline study participant parameters. Parametric tests were used to analyze parametric data if it passed the tests of normality; if it failed then non-parametric tests were used for analysis. Chi-square test was used to analyze categorical data. Pearson correlation test was used for parametric correlation analysis. The level of significance was set at $5 \%$. All $\mathrm{p}<0.05$ were considered significant. The data entry and analysis was performed using the MS Excel and GraphPad softwares.

\section{RESULTS}

A total of 524 patients were included in our study between age group of 12-80 years, mean \pm SD being $32.05 \pm 12.2$. $65 \%$ of the patients were from age group 2140 years (Figure 1). In our study the sex distribution observed was $181(34.5 \%)$ were females and $343(65.5 \%)$ were males (Figure 2). The most common clinical presentation being tinea cruris et corporis, followed by tinea cruris et corporis et faciei and tinea corporis et cruris (Figure 3). On the basis of dermatological examination, we found that the severity of the symptoms decreased drastically after giving oral Itraconazole $100 \mathrm{mg}$ BD for 6 weeks. The pretreatment severity score was $7.7 \pm 1.1$ and post treatment was $0.25 \pm 0.43$, indicating the excellent efficacy of the drug (Table 1). 21\%, 6.9\%, 6.5\%, $2.8 \%$ patients showed rise from the normal value in eosinophils, SGOT, SGPT, RBS, respectively. There was no evidence of increase in incidence of dermatophytosis in patients having RBS more than $200 \mathrm{mg} / \mathrm{dl}$ (Table 2). The pretreatment and post treatment comparison of liver function test parameters showed statistically significant difference in only SGOT with $\mathrm{p}$ value (paired $\mathrm{T}$ test) (Table 3).

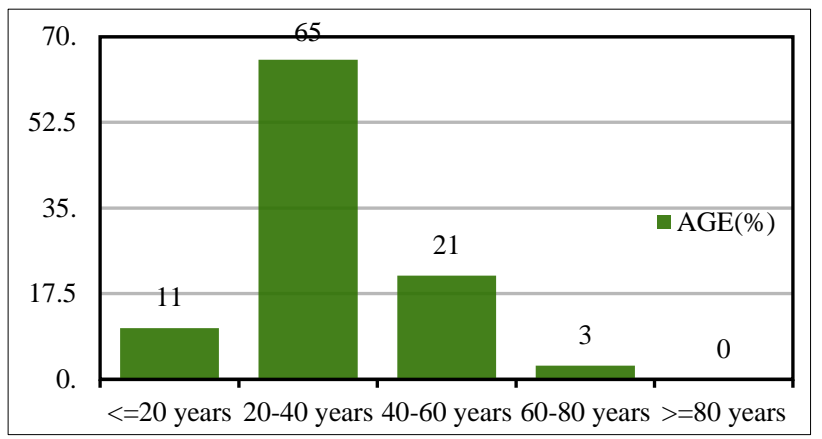

Figure 1: Age group in extensive tinea. 


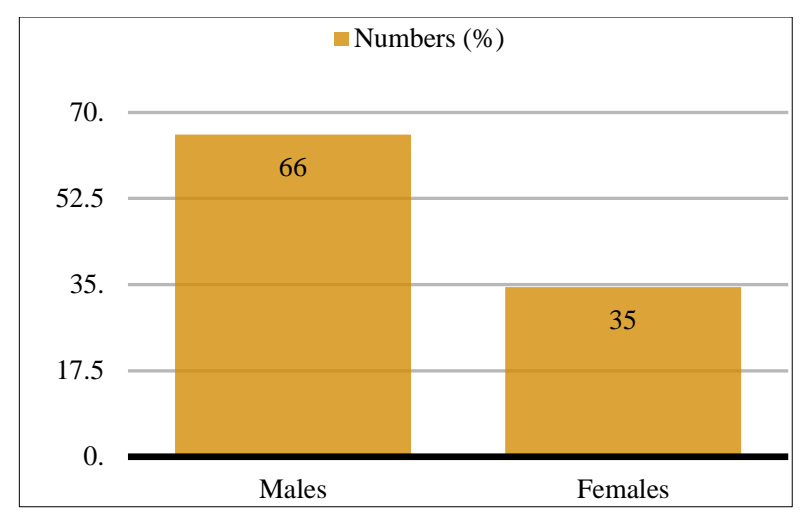

Figure 2: Sex distribution in extensive tinea.

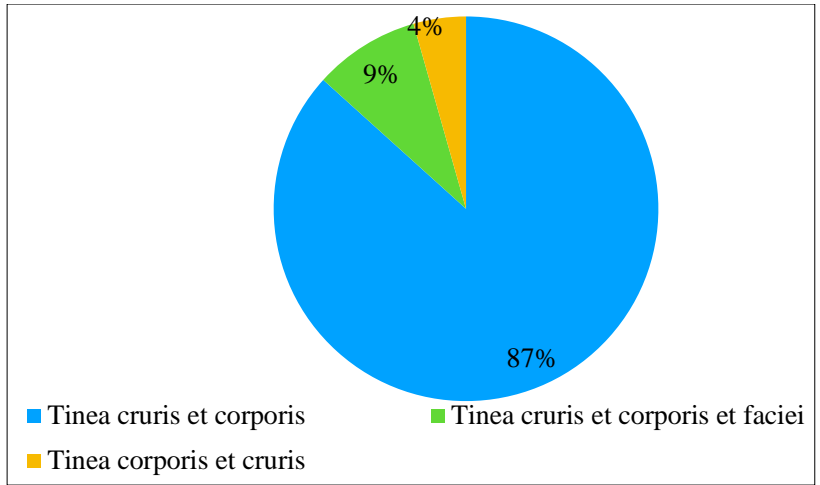

Figure 3: Pattern of distribution in extensive tinea.

Table 1: Clinical grading (scaling, itching, erythema) for extensive tinea on basis of severity.

\begin{tabular}{|c|c|c|c|c|}
\hline \multirow{6}{*}{ 1st visit } & \multicolumn{4}{|l|}{ Clinical grading } \\
\hline & Severity grading & No. of patients & Number (\%) & Mean \pm SD \\
\hline & 9 & 147 & 28 & \multirow{4}{*}{$7.7 \pm 1.1$} \\
\hline & 8 & 183 & 35 & \\
\hline & 7 & 105 & 20 & \\
\hline & 6 & 89 & 17 & \\
\hline \multirow{3}{*}{ 2nd visit } & 5 & 201 & 38 & \multirow{3}{*}{$4.06 \pm 0.82$} \\
\hline & 4 & 168 & 32 & \\
\hline & 3 & 155 & 30 & \\
\hline \multirow{2}{*}{$3^{\text {rd }}$ visit } & 0 & 404 & 77.1 & \multirow{2}{*}{$0.25 \pm 0.43$} \\
\hline & 1 & 120 & 22.9 & \\
\hline
\end{tabular}

Table 2: Baseline laboratory parameters.

\begin{tabular}{|ll|}
\hline Parameter & Number $(\%)$ \\
\hline WBC (>11,000) & 10.5 \\
\hline RBS( $>\mathbf{2 0 0} \mathbf{~ m g / d l )}$ & 2.3 \\
\hline Eosinophils $(>\mathbf{6 \%})$ & 21 \\
\hline Sr. Creatinine (>1 $\mathbf{~ m g / d l )}$ & 8.2 \\
\hline Total bilirubin (1.2 $\mathbf{~ m g / d l )}$ & 3.2 \\
\hline SGOT (>40 $\mathbf{~ m g / d l ) ~}$ & 6.9 \\
\hline SGPT $(>\mathbf{4 5} \mathbf{~ m g / d l )}$ & 6.5 \\
\hline
\end{tabular}

Table 3: Comparison of baseline post- treatment LFT.

\begin{tabular}{|ll|ll|}
\hline Test & Pre-treatment $($ Mean \pm SD) & Post- treatment $($ Mean \pm SD) & P value (Paired T test) \\
\hline Total bilirubin & $0.43 \pm 0.25$ & $0.45 \pm 0.40$ & 0.05 \\
\hline SGOT & $22.46 \pm 7.3$ & $23.54 \pm 10.8$ & 0.0003 (significant) \\
\hline SGPT & $21.42 \pm 10.82$ & $22.75 \pm 15.13$ & 4.00 \\
\hline
\end{tabular}

\section{DISCUSSION}

Itraconazole, a systemic antifungal agent is efficiently used in the treatment of superficial and deep mycoses. It inhibits the fungal cytochrome P450 dependent enzyme and thus impaires the conversion of lanosterol to ergosterol. The effect of itraconazole on cytochrome P450 is less as compared to other azoles, suggesting its less effect on human steroidogenesis, while more affinity towards fungi. Therefore, hepatotoxic reactions have rarely been reported with itraconazole. Adverse reactions to itraconazole includes drug reactions, gastrointestinal upset (nausea, abdominal pain, constipation), headache, dizziness, thrombocytopenia, gynecomastia, reversible edema of the extremities and metabolic side effects like hypokalemia, and hypertriglyceridemia. ${ }^{4}$ The level of hepatic transaminases increases in about 1\%-5\% of patients who have received continuous therapy with systemic itraconazole. ${ }^{4,5}$ Clinical hepatitis rarely occurs in patients and, recovery generally ensues with the cessation 
of medication. ${ }^{6}$ The recovery period varies from patients to patients, as reported in different literatures.

The present study was conducted in outpatient department of dermatology department of a tertiary care teaching hospital in Navi Mumbai. The demographic finding of the present study is showing high prevalence in males $(65.5 \%)$ compared to that of females $(34.5 \%)$, which could be because of excessive outdoor activities and perspiration. In the previous study conducted by Teklebirhan et al, majority of the population were female $(68.2 \%) .^{7}$ In our study, the maximum number of patients were from $21-40$ years of age $(65.3 \%)$. This is in accordance with study conducted by Poyyamozhi et al. ${ }^{8}$ In our study, we have observed that most of the patients presented with tinea cruries et corporis $(86.6 \%)$, followed by tinea cruris et corporis et faciei $(8.9 \%)$ and tinea corporis et cruris (4.4\%). Interestingly, among the current study population, tinea faciei was seen in adults patients, a location which has been described in children, and almost invariably this presentation could be because of over the counter topical steroid abuse. ${ }^{9}$ On further evaluation, on the basis of pre-treatment and posttreatment symptoms and dermatological examination i.e. itching, erythema, scaling, the patients were graded as 0 , $1,2,3$ respectively ( 0 - none, 1 - mild, 2 - moderate and 3severe); the highest score being 9 and the lowest being 0 . All the symptoms were between grade 6-9 before treatment and reduced to $0-1$ after treatment with oral Itraconazole $100 \mathrm{mg}$ BD for 6 weeks. The $\mathrm{p}$ value in comparison of 1 st and 3rd visit is 0.00 , which is highly significant indicating the efficacy of the drug. The post treatment itching could be the result of perspiration as there were no signs of activity of dermatophytosis on dermatological examination. In present study, the baseline data shows eosinophilia $(>6 \%)$ in $21 \%$ of our patients, which indicates that the dermophytosis infection could be more common in allergic patients as compared to normal individual. Due to the disruption of normal barrier function of skin in atopic dermatitis, the infection is chronic and more pronounced in patients with eosinophilia. A study conducted by Hay, Jillson, Huppert, Jones et al shows high incidence of allergic history with chronic dermatophytosis. ${ }^{10-12}$ We have observed higher WBC count $(>11,000)$ in $10.5 \%$ and raised RBS $(>200$ $\mathrm{gm} / \mathrm{dl}$ ) in $2.3 \%$ of study population. In the study by Evans et al, which compared non diabetic with diabetic patients, no significant difference was observed between both population group. This indicates that diabetes is not a major risk factor in etiology of dermatophytosis. ${ }^{13}$ In the given study, the effect of itraconazole $100 \mathrm{mg}$ BD for 6 weeks was seen in patients with extensive dermatophytosis on hepatic function. In our study, we have noted that $6.9 \%$ of the patients had shown raised SGOT. We have compared the baseline SGOT $(22.46 \pm 7.3)$ with that of post treatment SGOT $(23.54 \pm 10.8)$, which is statistically significant (0.0003). We have also noted that $6.5 \%$ of the patients had shown raised SGPT, on further comparing baseline SGPT $(21.42 \pm 10.82)$ to that of post treatment SGPT
(22.75 \pm 15.13$)$, but the difference was statistically insignificant (4.00). As per our findings, baseline total serum bilirubin was $0.43 \pm 0.25$ and post treatment was $0.45 \pm 0.40$, which is statistically not significant ( $\mathrm{p}$ value 0.05 ; Paired $\mathrm{t}$ test). We have not seen any patient developing clinical hepatitis after the oral therapy as mentioned above. In the previously various studies conducted by Boaz et al and Tucker et al to see the hepatotoxic effect of itraconazole, it was linked with dose and duration of the therapy. ${ }^{14}$ In our study, the dose and the duration was constant for all the patients.

\section{CONCLUSION}

The baseline and post treatment liver function test is important to monitor if the patient is on higher dose and longer duration of itraconazole therapy. The screening for high risk patients like poor liver function test, history of alcoholism, history of liver disease should be taken before stating the therapy.

\section{Funding: No funding sources}

Conflict of interest: None declared

Ethical approval: The study was approved by the Institutional Ethics Committee

\section{REFERENCES}

1. Sahoo AK, Mahajan R. Management of tinea corporis, tinea cruris, and tinea pedis: a comprehensive review. Indian Dermatol Online J. 2016;7(2):77-86.

2. Lou HY, Fang CL, Fang SU, Tiong C, Cheng YC, Chang CC. Hepatic failure related to itraconazole use successfully treated by corticosteroids. Hepat. Mon. 2011;11:843-6.

3. García Rodríguez LA, Duque A, Castellsague J, Pérez-Gutthann S, Stricker BH. A cohort study on the risk of acute liver injury among users of ketoconazole and other antifungal drugs. Br J Clin Pharmacol. 1999;48:847-52.

4. Amichai B, Grunwald MH. Adverse drug reactions of the new oral antifungal agents--terbinafine, fluconazole, and itraconazole. Int $\mathrm{J}$ Dermatol. 1998;37(6):410-5.

5. Persat F, Schwartzbrod PE, Troncy J, Timour Q, Maul A, Piens MA. Abnormalities in liver enzymes during simultaneous therapy with itraconazole and amphotericin B in leukaemic patients. J Antimicrob Chemother. 2000;45(6):928-9.

6. Hann SK, Kim JB, Im S, Han KH, Park YK. Itraconazole-induced acute hepatitis. Br J Dermatol. 1993;129(4):500-1.

7. Teklebirhan G, Bitew A. Prevalence of Dermatophytic Infection and the Spectrum of Dermatophytes in Patients Attending a Tertiary Hospital in Addis Ababa, Ethiopia. Int $\mathbf{J}$ Microbiol. 2015;2015:653419.

8. Poyyamozhi JS. Prevalence and factors influencing anaemia among urban adolescent females, a cross 
sectional study. Int J Community Med Public Health. 2018;5(4):1354-9.

9. Panda S, Verma S. The menace of dermatophytosis in India: The evidence that we need. Indian J Dermatol Venereol Leprol. 2017;83:281-4.

10. Hay RJ. Chronic dermatophyte infections. I. Clinical and mycological features. $\mathrm{Br} \mathrm{J}$ Dermatol. 1982;106:1-7.

11. Jillson OF, Huppert M. The immediate wheal and 2448 hour tuberculin type edematous reactions to trichophytin. J Investig Dermatol. 1949;12:179-85.

12. Jones HE, Reinhardt JH, Rinaldi MG. A clinical, mycological, and immunological survey for dermatophytosis. Arch Dermatol. 1973;108:61-65.

13. Evans SL, Nixon BP, Lee I, See D, Mooradian AD. The prevalence and nature of podiatry problems in elderly diabetic patient. J Am Geriatr Soc. 1991;39:241-5.

14. Tucker RM, Haq Y, Denning DW, Stevens DA. Adverse events associated with itraconazole in 189 patients on chronic therapy. J Antimicrob Chemother. 1990;26:561-6.

Cite this article as: Thakare V, Patel NK, Patil S, Modi N. Oral antifungal: the safety and efficacy of oral itraconazole in dermatophytosis. Int J Basic Clin Pharmacol 2019;8:2470-4. 